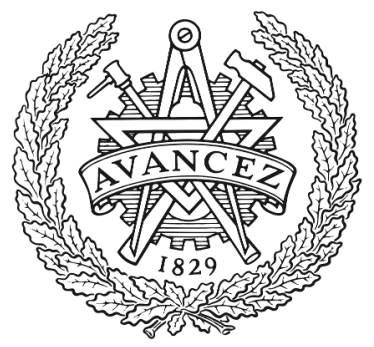

CHALMERS

UNIVERSITY OF TECHNOLOGY

\title{
Key aspects of digital image correlation in impact tests of reinforced concrete beams
}

Downloaded from: https://research.chalmers.se, 2023-04-25 23:43 UTC

Citation for the original published paper (version of record):

Johansson, M., Rempling, R., Sanz, G. et al (2019). Key aspects of digital image correlation in impact tests of reinforced concrete beams. IABSE Symposium, Guimaraes 2019: Towards a Resilient Built Environment Risk and Asset Management - Report: 961-968

N.B. When citing this work, cite the original published paper. 


\title{
Key aspects of digital image correlation in impact tests of reinforced concrete beams
}

\author{
Morgan Johansson, Rasmus Rempling \\ Dept. Civil \& Environmental Eng., Chalmers University of Technology, Gothenburg, Sweden
}

Gonzalo S.D. de Ulzurrun*, Carlos Zanuy

Dept. Continuum Mechanics \& Structures, Universidad Politécnica de Madrid, Spain

Contacting author: g.ulzurrun@caminos.upm.es (orcid.org/0000-0002-6733-1555)

\begin{abstract}
This paper studies 2-D high speed photography combined with digital image correlation (DIC) applied to experimental research of reinforced concrete beams at moderate loading rates. The aim of the present research is to understand the influence of 2-D DIC set-up parameters in the results. Drop-weight tests have been completed in $1180 \times 100 \times 100 \mathrm{~mm}$ longitudinally reinforced concrete beams. The study has confirmed results sensitivity to image subdivision and mesh properties. While smaller subdivision sizes allow to obtain results nearby boundaries, being more suitable to study local effects, larger sizes enhance computational cost, increase mesh stability and accuracy. A discussion of key aspects of 2-D DIC for measuring different parameters (such as acceleration, displacements, strains and strain-rate) is presented along this paper.
\end{abstract}

Keywords: impact; digital image correlation; beams; concrete; sensitivity; facet.

\section{Introduction}

Hazardous events and antagonistic activities are part of our daily challenging society. The need for understanding the response of impact-loaded concrete structures has increased for the last years. These structures have shown high sensitivity to develop brittle failure when subjected to impact loading $[1,2]$.

Numerous experimental investigations on the impact performance of reinforced concrete (RC) have been carried out by different authors [3-5], with purpose of increasing the knowledge on concrete dynamic behaviour. Valuable cinematic measurements might be found among scientific literature, such as accelerations, displacements or strain at representative discrete points, as well impact force, support reactions or dissipated energy. Nevertheless, it is unclear how the distribution of the former parameters evolves over the surface of concrete elements when subjected to impact loading. Consequently, there is a lack of generalized understanding of some relevant dynamic effects, such as the propagation of impact loads along the tested element $[6,7]$, the distribution of inertia forces $[4,8]$ or the strain-rate effects at the failure mechanism [9].

An interesting way to study these effects is the use of optical full-field measurement methods. This technique might be divided into two main groups, interferometric methodologies and noninterferometric techniques, according to their 
approach to measure deformations with light. While the former focuses on phase difference of light wave from a coherent light source, the latter compares changes on a grey-scale pattern with a simple set-up and post-processing. Among the noninterferometric methods, digital image correlation (DIC) stands out as widespread tool for experimental measurement due to its robustness, ease of implementation and adaptability for different test setups [10-13]. Furthermore, DIC in combination with high speed photography (HSP) has been proven a reliable technology to measure dynamic tests using 2-D imaging $[6,14]$, as well as 3-D [15, 16]. Nevertheless, its application in impact loading tests on RC beams is still limited in the scientific literature, with a further need to establish guidelines for its future use.

The aim of the research presented in this paper is to give advice on the use of DIC when conducting impact tests on RC beams. Hence, the influence of test set-up and post-processing settings on results will be discussed from a critical perspective.

The study will focus on three impact test carried out at Chalmers University of Technology, Sweden, that have been analysed with 2-D DIC. The tests presented are part of a broader experimental campaign carried out by Jönsson \& Stenseke [17]. The results of these tests are analysed with a different perspective in Ulzurrun et al. [18], exploring the potential of using this technology to analyse impact tests.

\section{Experimental research}

\subsection{Test description}

The experimental campaign covers three prismatic $\mathrm{RC}$ beams tested under impact loading. The tests were recorded with a HSP camera and analyzed using DIC measurement technique. An overview of the test set-up is shown in Figure 1.

Beams, that were identical, were named numerically, from 12 to 14 . The dimensions of the beams were $1180 \mathrm{~mm}$ of length with a square cross-section with a side of $100 \mathrm{~mm}$. A reinforcement ratio $\rho=0.7 \%$ was chosen, without placing any transversal reinforcement. Tests were completed in a three-point bending configuration, with a span length of $1000 \mathrm{~mm}$ between supports. Both supports consisted of a solid steel roll with a diameter of $80 \mathrm{~mm}$ that was not intended to prevent the uplift movements upon impact loading. A speckle pattern was applied on one side of the beam. Pattern size ranged from 1.5 to $3.5 \mathrm{~mm}$, still recognisable for image resolution employed whilst keeping contrast with neighbouring pixels.

A free falling $20 \mathrm{~kg}$ weight was dropped onto the beam's midspan. The weight consisted of a solid steel cylinder of $80 \mathrm{~mm}$ diameter and $500 \mathrm{~mm}$ height, with a spherical impact surface of $200 \mathrm{~mm}$ radius. The mass was released from a height of $5 \mathrm{~m}$, using a drop weight testing rig, reaching an impact velocity of $9.9 \mathrm{~m} / \mathrm{s}$. A speckle pattern was also applied on the side faces of the drop-weight.

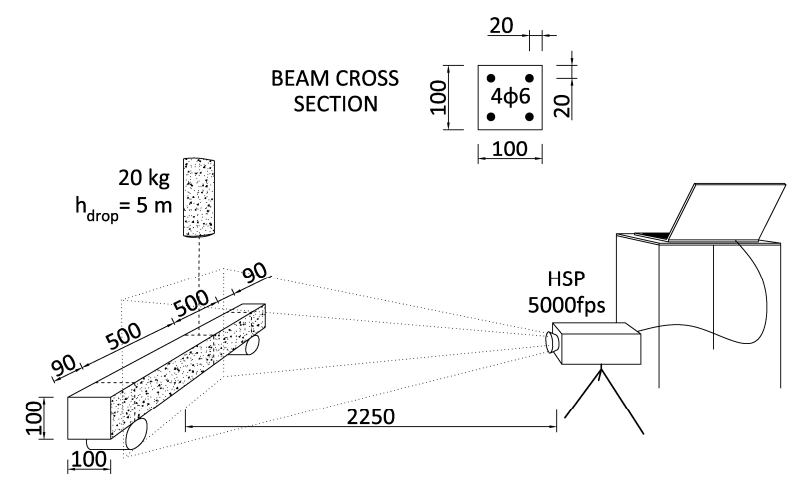

Figure 1. Schematic test set-up. Dimensions in $\mathrm{mm}$.

\subsection{Measurement system:DIC}

Image acquisition was done using a single HSP camera. 2-D imaging was employed as it was not expected any deformations out of the vertical plane. The employed lens, Tamron AF 28-75/2.8, was mounted in a Photron SA4 camera. The focal length of the lens was set to $75 \mathrm{~mm}$ and its aperture to $f / 2.8$. The camera body was placed perpendicular to the lateral surface of the beam, at $2225 \mathrm{~mm}$ of distance. Images were recorded at 5000 frames per second (fps), with a shutter speed of $1 / 5000$. Due to high recording speed natural light illumination was insufficient, thus an additional white light source was employed during the tests. The image resolution was $1024 \times 512$ pixels, covering a $626.30 \times 313.15 \mathrm{~mm}^{2}$ area. Pixel size, $0.61162 \mathrm{~mm}$, was obtained by calibrating the spatial scale with a $60 \mathrm{~mm}$ gauge block.

The captured images were analysed with DIC. This technique is capable of tracking a grey-scale 
pattern, comparing it with a reference stage. The software obtains the results through virtual divisions of the image in square subimages, which are more capable to be uniquely identified than an individual pixel [10]. These subimages are commonly known as facets or subsets. An individual facet forms a single point of measurement, but together with other facets might form an equally spaced mesh, able to generate full-field measurements. The distance between facets in a mesh is known as point distance or step size, which can also be expressed as its ratio to the facet size (distance-to-size ratio).

Once the deformation has begun each facet is correlated with its undeformed image through a speckle pattern, which is the carrier of surface deformation information. According to Dong et al. [13] a good speckle pattern should fulfil the following requisites: high contrast, randomness, isotropy and adherence stability. Since a concrete surface is generally monochrome an artificial speckle pattern should be provided. Some authors have applied a pattern by distributing randomly black or white paints over the object surface [6, 14]. An interesting way to apply the paint to create a proper pattern might be by stamping the paint with a natural sea sponge.

Tested beams presented in this study were provided an artificial speckle. The paint, that was latex based, was applied with a natural sea sponge as random black flecks over a white background coating. The resulting pattern at the recorded area for all beams is shown in Figure 2. In order to enhance the spatial resolution of the image, only a part of the beam was recorded. The field of view covered the impact area at midspan as well as almost half the beam length, as is represented schematically in Figure 1 (symmetry is taken in advantage).

The software used to analyze the test was GOM Correlate. Meshes and individual facets have been used in the analysis. Individual facets were used to obtain displacements, velocities or accelerations. Meshes provided full-field data of the previous parameters, in addition to strains, and their rates. This software generates meshes that arrange facets in a triangular layout, with a constant distance between them.
Different facet sizes and distance-to-size ratios were employed to analyze the results. The facet size employed ranged between 4 and 32 pixels, increasing geometrically by a factor of 2 . While distance-to-size ratios employed were $1 / 8,2 / 8$, $3 / 8,4 / 8$ and $6 / 8$, except for the 4-pixel meshes that the ratios employed were $2 / 8,4 / 8$ and $6 / 8$.

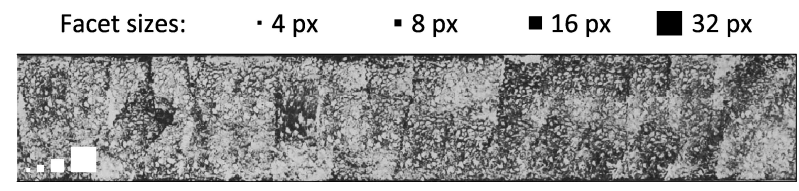

Beam 12

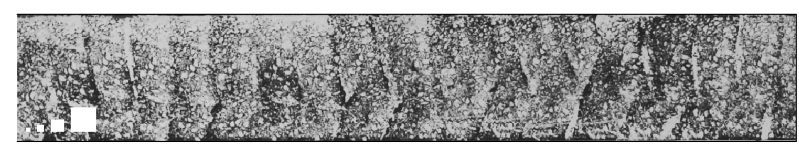

Beam 13

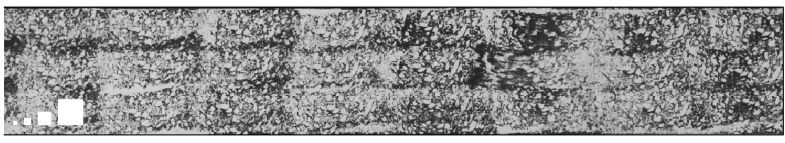

Beam 14

Figure 2. Recorded speckle pattern of each beam compared with the studied facet sizes.

\subsection{Experimental results}

The typical evolution of the measured parameters at Beam 12 is shown in Figure 3 for different facet sizes. The other two tests showed similar results. The results are shown in terms of midspan deflection and drop-weight vertical acceleration, as well as major strain and its rate at the midspan top fiber. The two former parameters were measured with individual facets, while the two latter were measured with facet meshes, with a constant distance-to-size ratio of $4 / 8$.

The displacement shows a good agreement for different facets sizes, providing almost identical results for the two larger facet sizes. The maximum deflection was reached $18 \mathrm{~ms}$ after the beginning of the impact load with a value of almost $60 \mathrm{~mm}$. The drop-weight acceleration history presents higher noise levels for smaller facet sizes, while larger facet results are steadier. There are two stages, the first one having a high peak acceleration with a very short duration while the second one had a smaller peak load. The major strain recorded at the midspan top fiber show large noise levels once the maximum deflection is reached, presenting a high strain 
(a)

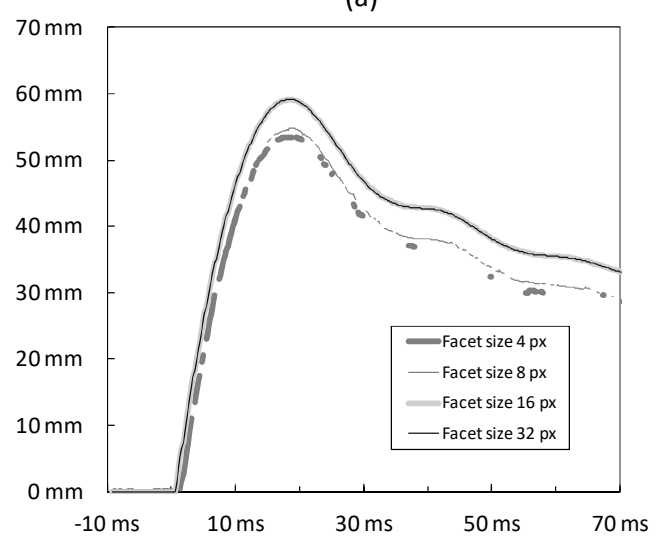

(c)

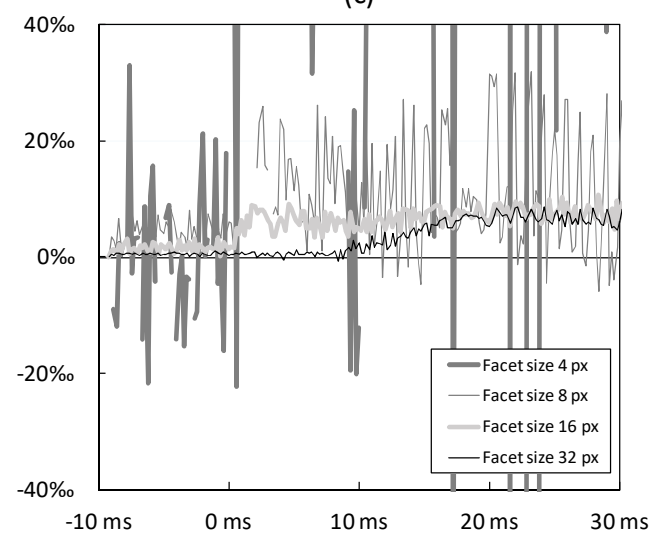

(b)

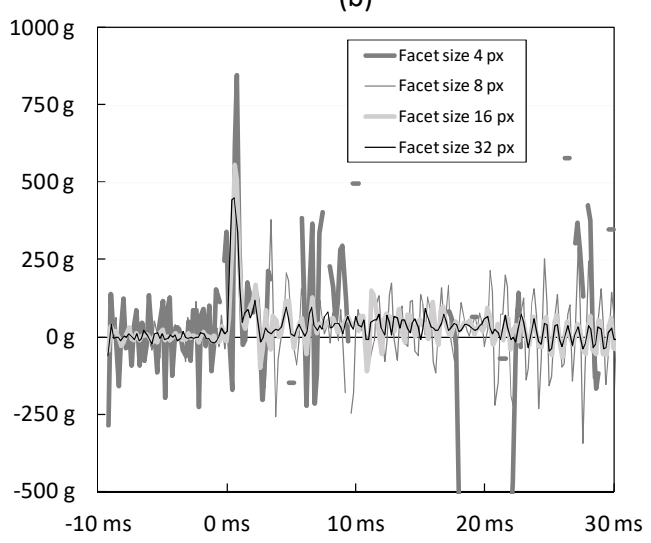

(d)

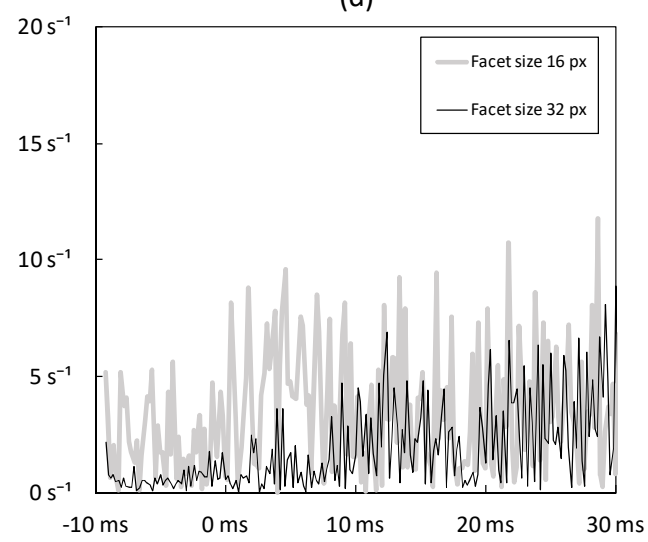

Figure 3. Beam 12 DIC measurements over time for different facet sizes: a) midspan deflection; b) dropweight acceleration; c) top fiber midspan strain; d) top fiber midspan strain-rate.

value, $10 \%$. Strain-rates are only displayed for facet size of 16 and 32 pixels, due to the high noise levels of the other sizes. Nevertheless, noise levels for the displayed meshes are considerable high, especially for a facet size of 16 pixels. The values recorded for a facet size of 32 pixels once the impact has started range within the strain-rate domain for impact loading, 1-10 $\mathrm{s}^{-1}$. It should be noted that measurements for smaller mesh sizes shows gaps that correspond to uncomputed steps, due to the lack of facet stability for those sizes.

\section{Analysis of results and discussion}

The results presented in the previous section have confirmed their dependence on facet properties chosen in the DIC analysis, in terms of noise levels and facet stability. Facet stability is the capacity of a facet mesh to maintain its measurement points over time. The lack of facet stability responds to the inability to establish a correlation with the reference image. This might be due to noise, motion blur or monochromatic areas within the speckle pattern.

An interesting way to study the influence of facet properties on stability is to measure over time the percentage of facets that are correlated with the reference image. This analysis has been done for different meshes (according to the facet size and distance-to-size ratios referred-to in Section 2.2). The analysis has shown that for a certain facet size the distance-to-size ratio had a small influence, increasing slightly the stability for smaller ratios. The facet stability is presented in Figure 4 as the average percentage of measuring points correlated for each facet size. It can be noted that very small facets sizes, such as 4 pixels, may be too instable, even showing a loss of measurement points before impact occurs. This might be due to the fact that smaller facet sizes cover lesser variations of greys, being harder to identify uniquely. In addition, the 4-pixel facet size is somehow similar to the size of the speckle pattern, as it is shown in Figure 2, providing smaller colour gradients. Larger facet 
sizes were rather stable, showing smaller drops of measurement point points, once the impact began, as facet sizes were larger.

\subsection{Sensitivity analysis for facet properties}

Some measurements presented in Figure 3 shows large fluctuations even before impact occurs. This might be due to errors related to the imaging system, the set up or to the correlation algorithm [10]. An interesting way to characterize measurement resolution of a certain parameter is to study its standard deviation before deformation, suggested by Pritchard et al. [19]. This technique has been used here to perform a sensitivity analysis to facet properties of the measurements at each stage for the $10 \mathrm{~ms}$ previous to impact loading.

A Figure 5 shows a study of the measurement resolution for different parameters. In each graph is plotted the standard deviation of each measured parameter for a certain facet size. The results are

(a)

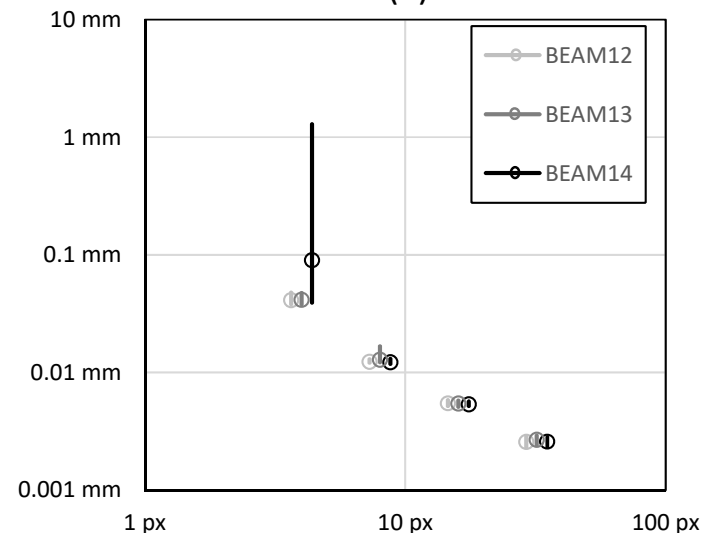

(c)

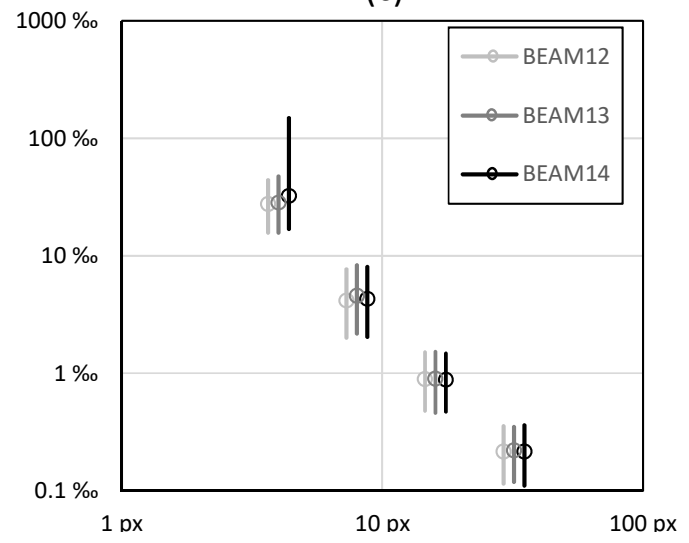

shown in terms of maximum, minimum and average value of the standard deviation obtained at different stages and covering different distanceto-size ratios (referred to in Section 2.2). It is noteworthy that studied parameters, namely

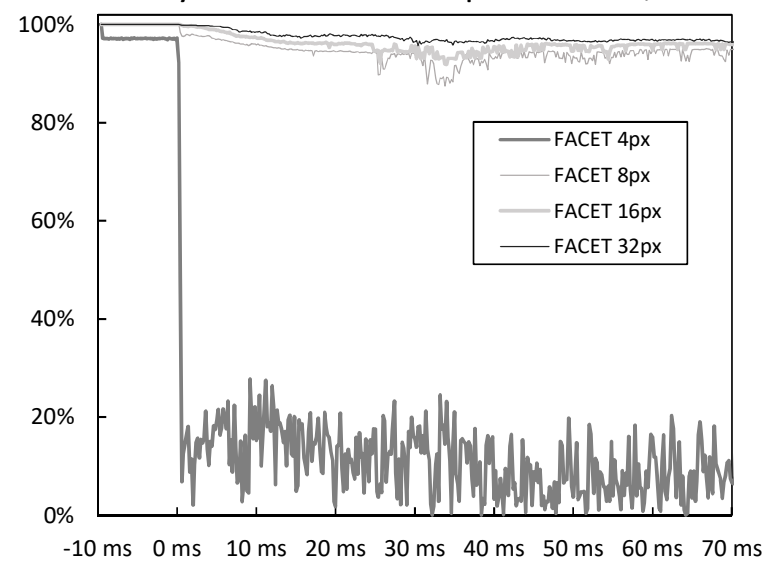

Figure 4. Beam 12 percentage of facets correlated, for different facet sizes, over time.

(b)

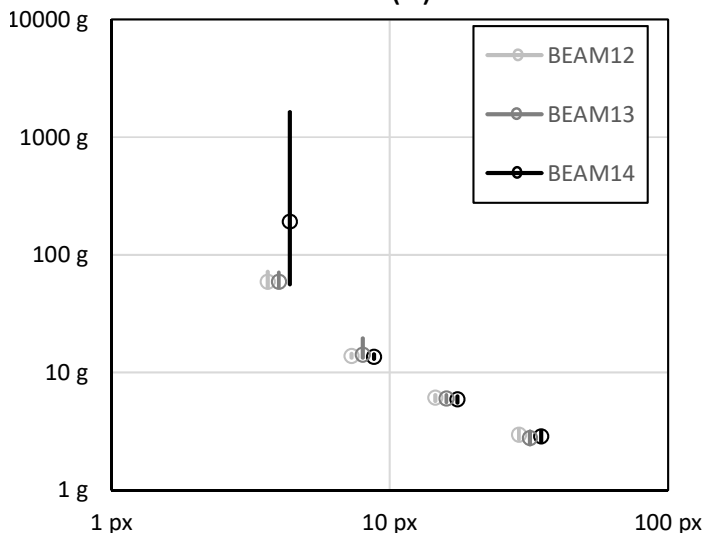

(d)

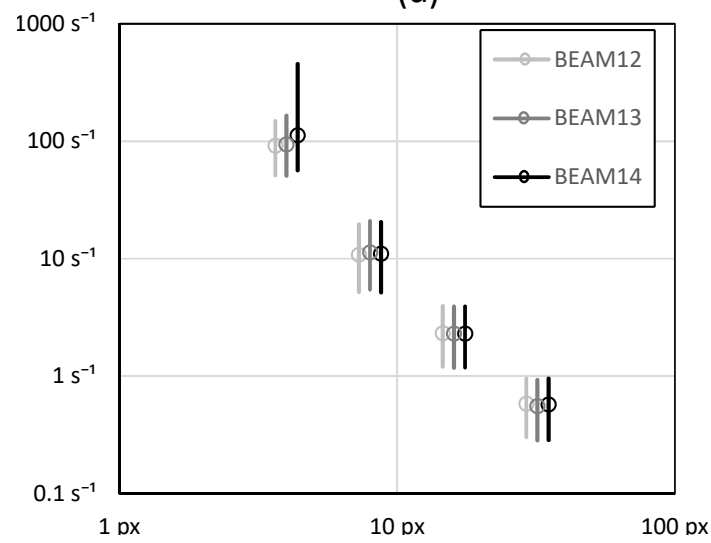

Figure 5. Sensitivity analysis. Maximum, minimum and average value of standard deviation, before beams deformation, for different facet sizes: a) vertical displacement; b) vertical acceleration; c) principal strain; d) principal strain-rate. 
displacement, acceleration, principal strain and its rate, have similar error distribution, but higher in magnitude for the parameters obtained by timedifferentiation, as numerical differentiation amplifies the noise included in any measurement. Besides, Beam 14 has shown for all parameters large errors for a 4-pixel facet size, probably due to the speckle pattern of this beam, with few smeared areas.

In general, vertical displacements and accelerations showed a consistent standard deviation distribution, with similar maximum and minimum values. Both increased its resolution with larger facet sizes, ranging from 0.003 to $0.5 \mathrm{~mm}$ and 2.5 to $70 \mathrm{~g}$, respectively. Principal strain and its rate standard deviations were more dispersed, with average value equidistant between them. The reason of this is the influence of the distance-tosize ratio, as it is shown in Figure 6, where the resolution is defined for each of meshes employed. It can be observed that higher distance-to-size ratios decreased resolution. Strains are computed as a numerical differentiation of the displacement field in the spatial domain, therefore errors are smoothened with larger point distance. Although not shown in this paper, similar results were observed for the principal strain-rate. Principal strain and its rate resolution ranged from 0.1 to $50 \%$ and 0.3 to $170 \mathrm{~s}^{-1}$, respectively.

\subsection{Discussion}

It is clear from the previous analysis that facet properties, size and distance-to-size ratio, play a key role in the resolution of DIC measurements. Nevertheless, decreasing noise levels by changing facet properties may have some downsides. An increase of facet size increases measurement resolution. Although it reduces the capacity to measure near to the boundaries, as it sets-back the limits of the mesh from beam boundaries. Additionally it increases computing cost, if point distance is kept constant. Similarly, when measuring strains, or its rate, an increase of distance-to-size ratio could enhance resolution, as well as decreasing computational costs. Nevertheless, this increase lowers measurement point density, therefore smoothening or even covering local effects.

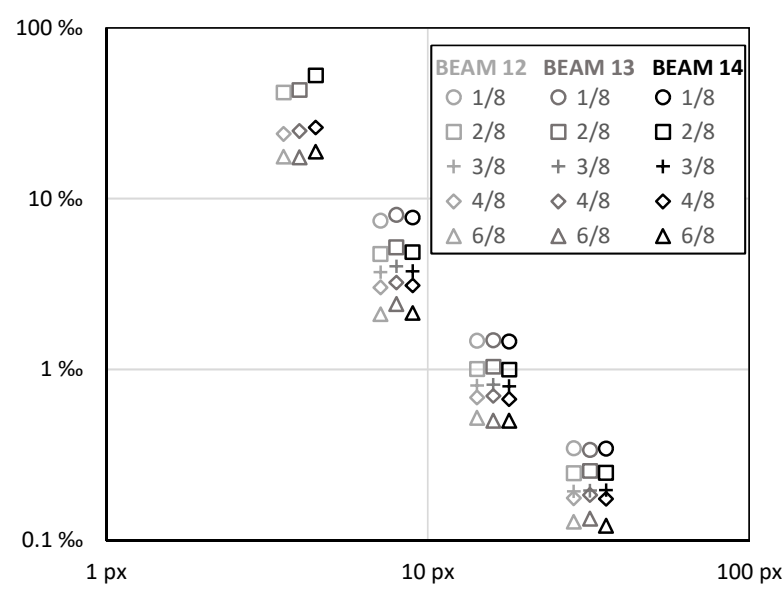

Figure 6. Principal strain resolution. Standard deviation, for the undeformed beam in terms of facet size, for different distance-to-size ratios.

It seems that some criteria have to be set to enhance resolution by choosing ideal facet properties. By comparing the standard deviation values for the undeformed beams, shown in Figures 5 and 6 , with the expected measured values some guidelines might be established. In addition, stability should also be taken into account, and therefore further analysis of these tests should avoid 4 pixels facets.

Typical deflections in impact test are in the range from a few millimetres to more than $100 \mathrm{~mm}$. The resolution for all the meshes employed were well under those values, therefore any of the employed meshes, is suitable to be used when studying the midspan deflections. In terms of vertical accelerations, the expected values are in the range from almost 100 to $1000 \mathrm{~g}$. Nevertheless, if the post-peak wave needs to be analysed, facet sizes should be at least 16 pixels or larger. However, as the acceleration is a very sensitive parameter the use of the largest facet size possible is strongly recommended. Regarding principal strains, the maximum expected value for concrete is $5 \%$ in compression and $0.2 \%$ in tension, before cracks propagate. Those strains compared with the resolution of the meshes seem too small to be distinguished from the noise, except for 32-pixel facet size meshes with large distance-to-size ratios. Lastly, strain-rates within the impact domain range between 1-10 $\mathrm{s}^{-1}$, which compared to the sensitivity analysis, seem to be also too high to obtain reasonable measurements. 
Another use of principal strain measurements may be to study the localization of cracks, not visible through other means, which produce tensile strains artificially high. Figure 7 shows crack patterns for beam 12 at the same instant, when using different facet settings. Scale range has been adjusted taking into account the resolutions showed in Figure 6, in order to avoid noise to be included in the crack patterns. These patterns show how spurious data decrease by increasing facet size and distance-to-size ratio, but also that details are lost as data smoothens. Therefore, medium facet sizes and distance-to-size ratios is recommended to be used to localise cracks accurately. It is noteworthy that the loss of measurement points in the vicinity of cracked areas may explain part of the small decreases of stability observed in Figure 4.

$16 p x$ facets at $2 p x$

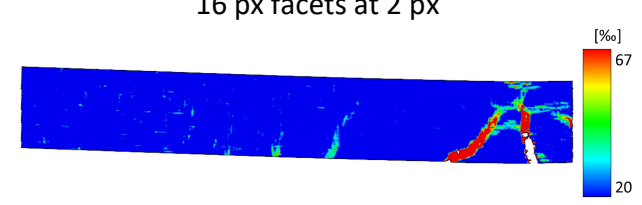

$16 \mathrm{px}$ facets at $12 \mathrm{px}$

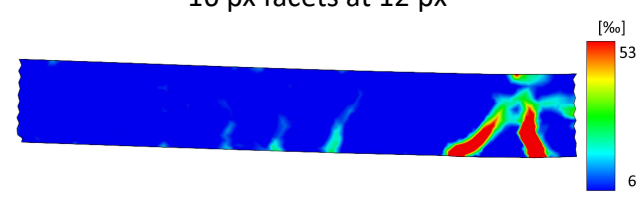

$32 p x$ facets at $4 p x$

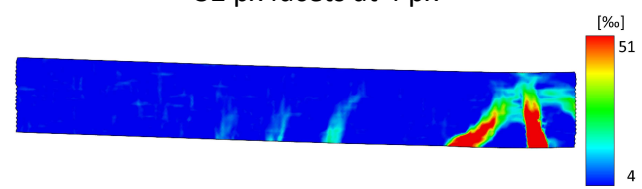

$32 \mathrm{px}$ facets at $24 \mathrm{px}$

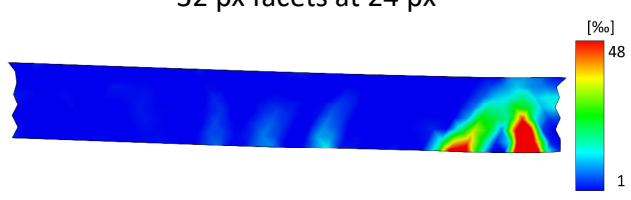

Figure 7. Beam 12 crack pattern 2,6 ms after impact for different meshes.

It has been observed that some measured parameters present low resolution levels for any of the facet properties studied. The resolution might be increased by different means. A common way to increase the measurement quality might be to improve picture resolution. Nevertheless, in order to achieve best results speckle size should be adapted to the resolution of the camera, by reaming large enough to be pictured sharply, but small enough to provide a wide grey variety to the pattern within the facet. Besides, some authors
$[10,13]$ have stated that entropy and high colour gradients in speckle pattern improves results.

Another interesting way to enhance measurement is by increasing the frame rate, while keeping a similar picture resolution. This would increase the samples recorded in brief events, such as the impact acceleration. Nevertheless, if noise levels are kept constant, higher frame rates might increase error for derived parameters due to the smaller time step in the numerical differentiation. Related to this, increases of shutter speed may reduce errors, as motion blur decreases for short exposure times. Motion blur is the distortion of a photographed object, produced by its movement during the exposure time. For instance, the dropweight at impact speed would move $1.2 \mathrm{~mm}$ during this period, i.e. equivalent to 2 pixels, with a shutter speed of $1 / 5000 \mathrm{~s}$. This results in a distortion of the image that may lead to higher noise levels. In addition it also explains the noise levels observed in Figure 3, after impact occurs, and in Figure 7 near the supports, the noise is greater than those shown in Figures 5 and 6, computed under static conditions.

\section{Conclusion}

Experimental results of three RC beams tested at under impact load are presented in this paper. 2-DDIC has been employed as a full-field measurement system. The study analysed the influence of DIC set-up parameters and other error sources in the results, aiming to reduce noise levels and loss of measurement points.

The study shows that all parameters measured are sensible to facet, or sub-set, size, enhancing results quality with its increase. Point distance, or step size, only affected strains and strain rates, improving the resolution with its increase. Nevertheless the increase of both factors is translated into a lower accuracy of local effects. Therefore the ideal facet properties should be adjusted to its purpose, even changing from one parameter to another.

Other factors, related with experimental set-up, have influence in the accuracy of DIC measurements. Those have been discussed along the paper setting their advantages and drawbacks. 


\section{Acknowledgements}

A substantial part of this work was carried out during a research stay of the corresponding author at Chalmers University of Technology. The financial support provided by the following sources is gratefully acknowledged: Swedish Contingencies Agency, The Swedish Fortification Corp's Research Fund, Spanish Ministry of Economy (Project No. BIA2016-74960-R). In addition, the authors also thank "Fundación José Entrecanales Ibarra" for funding the PhD fellowship of the corresponding author. Also the authors would like to express their gratitude to Ph.D. Joosef Leppänen, Adj. Prof. Mathias Flansbjer and Sebastian Almfeldt at Chalmers University of Technology for kindly providing help with the experimental used in this study.

\section{References}

[1] Sharma H, Hurlebaus S, Gardoni P. Performancebased response evaluation of reinforced concrete columns subject to vehicle impact. Int J Impact Eng 2012; 43: 52-62.

[2] Schellenberg K. On the design of rockfall protection galleries. vdf Hochschulverlag an der ETH Zürich. Epub ahead of print 2009. DOI: 10.3929/ETHZ-A005816027.

[3] Delhomme F, Mommessin M, Mougin JP, Perrotin P. Simulation of a block impacting a reinforced concrete slab with a finite element model and a mass-spring system. Eng Struct 2007; 29: 28442852.

[4] Saatci S, Vecchio F. Effects of shear mechanisms on impact behavior of reinforced concrete beams. $A C l$ Struct J 2009; 106: 78-86.

[5] Ulzurrun GSD, Zanuy C. Enhancement of impact performance of reinforced concrete beams without stirrups by adding steel fibers. Constr Build Mater 2017; 145: 166-182.

[6] Isaac P, Darby A, Ibell T, Evernden M. Experimental investigation into the force propagation velocity due to hard impacts on reinforced concrete members. Int J Impact Eng 2017; 100: 131-138.

[7] Cotsovos DM, Stathopoulos ND, Zeris CA. Behavior of RC Beams Subjected to High Rates of Concentrated Loading. J Struct Eng 2008; 134: 1839-1851.
[8] Banthia N, Mindess S, Bentur A, Pigeon M. Impact testing of concrete using a drop-weight impact machine. Exp Mech 1989; 29: 63-69.

[9] Zanuy C, Ulzurrun G. Discussion of "Simplified Approach for Assessing Shear Resistance of Reinforced Concrete Beams under Impact Loads" (Paper by Wei-Jian Yi, De-Bo Zhao and Sashi K. Kunnath). ACl Struct J 2017; 114: 789-791.

[10] Pan B, Qian K, Xie H, Asundi A. Two-dimensional digital image correlation for in-plane displacementa and strain measurement: a review. Meas Sci Technol 2009; 20: 1-17.

[11] Cinar AF et al. An autonomous surface discontinuity detection and quantification method by digital image correlation and phase congruency. Opt Lasers Eng 2017; 96: 94-106.

[12] Kashfuddoja M, Prasath RGR, Ramji M. Study on experimental characterization of carbon fiber reinforced polymer panel using digital image correlation: A sensitivity analysis. Opt Lasers Eng 2014; 62: 17-30.

[13] Dong YL, Pan B. A Review of Speckle Pattern Fabrication and Assessment for Digital Image Correlation. Exp Mech 2017; 57: 1161-1181.

[14] Kirugulige MS, Tippur H V., Denney TS. Measurement of transient deformations using digital image correlation method and high-speed photography: application to dynamic fracture. Appl Opt 2007; 46: 5083-5096.

[15] Siebert T, Becker T, Spiltthof K, Neumann I, Krupka R. High-speed digital image correlation: error estimations and applications. Opt Eng 2007; 46: 17.

[16] Helfrick MN, Niezrecki C, Avitabile P, Schmidt T. 3D digital image correlation methods for full-field vibration measurement. Mech Syst Signal Process 2011; 25: 917-927.

[17] Jönsson J, Stenseke A. Concrete Beams Subjected to Repeated Drop-Weight Impact and Static Load. Chalmers TH, 2018.

[18] Ulzurrun GSD, Zanuy C, Johansson M, Rempling R. Impact propagation effects along reinforced concrete beams. In: Proceedings of the IABSE Symposium. Guimarães, 2019.

[19] Pritchard RH, Lava P, Debruyne D, Terentjev EM. Precise determination of the Poisson ratio in soft materials with 2D digital image correlation. Soft Matter 2013; 9: 6037-6045. 